\title{
Glomerulonephritis - Do We Have Promising Role of Monoclonal Antibodies?
}

\author{
Sonia Sharma ${ }^{1 *}$ and Sanjiv Saxena ${ }^{2}$ \\ ${ }^{1}$ Consultant Pediatrics Nephrology, Max Super-specialty and PSRI Hospital, Saket, New Delhi, India \\ ${ }^{2}$ Head of Nephrology Department, PSRI Hospital, Sheikh Sarai, Saket, New Delhi, India
}

*Corresponding author: Sonia Sharma, Consultant Pediatrics Nephrology, Max Super-specialty and PSRI Hospital, Saket, New Delhi, India; Tel: +91-8802954359; E-mail: sonyasharma01@gmail.com

Received date: November 24, 2016; Accepted date: December 19, 2016; Published date: December 22, 2016

Citation: Sharma S, Saxena S (2016) Glomerulonephritis - Do We Have Promising Role of Monoclonal Antibodies? J Clin Exp Nephrol 1: 23. DOI: $10.21767 / 2472-5056.100023$

Copyright: (C) 2016 Sharma S. This is an open-access article distributed under the terms of the Creative Commons Attribution License, which permits unrestricted use, distribution, and reproduction in any medium, provided the original author and source are credited.

\section{Abstract}

In present era, drugs like steroids, cytotoxic drugs and calcineurin inhibitors are the backbone to treat immune mediated glomerular diseases. Failure of these therapies often results in persistence of proteinuria, refractory illness and later chronic kidney diseases. The quests to find an answer to such diseases have resulted in the introduction of monoclonal antibodies or target specific drugs. Rituximab is the first monoclonal antibody, which was studied extensively in pauci-immune glomerulonephritis and is now approved for its role in refractory and new onset renal vasculitis. Belimumab has shown role in active systemic lupus erythematous (SLE). With increasing insight in glomerular pathophysiology, many new monoclonal antibodies are now being under trial. Their encouraging results allowed further research on target cytokines, inflammatory mediators and different lymphocyte populations. This review shall discuss monoclonal and their emerging role in glomerulonephritis.

Keywords: Monoclonal antibody; Proteinuria; Vasculitis; Glomerulonephritis

\section{Introduction}

Monoclonal antibodies can target immune mechanism either at antigen presenting cell, T-cell or B-cell pathway, complement system, co-stimulatory molecule or as an anticytokine therapy. Paul Ehrlich and Elie Metchnikoff were first one to give this idea of target molecule and received Nobel Prize in 1908 for their work on immunity and target immune mechanism [1]. Kohler and Milstein discovered the first monoclonal antibody against Bcell line in mice in 1975 and shared Nobel prize for physiology of medicine in 1984 [2]. Since the generation of first molecule and their magic role in malignancy, there is a rapid advancement in both number of monoclonal drugs and their trials for testing efficacy in other diseases. Glomerulonephritis (GN) is indeed one such diseases, where to prevent progression to chronicity, monoclonal drugs were used and showed significant improvement. OKT3 was the first monoclonal drug used in kidney transplant rejection and then numbers of monoclonal were introduced in transplantation. Subsequently these were then introduced in glomerulonephritis and showed their efficacy. This review shall focus update on exclusive role of monoclonal drugs, trials to clinical usage in glomerulonephritis.

\section{Rituximab}

In GN, rituximab is the first extensively used and successful monoclonal drug. It is now approved for ANCA vasculitis. It is a murine-human chimeric genetically engineered monoclonal antibody against $\mathrm{CD} 20$, an immature and mature $B$ cells surface marker, which is not expressed on plasma cells. Drug contains human IgG1 in the constant region ( $\mathrm{Fc}$ ) and murine heavy and light chain in variable regions (Fab).

It has an anti CD-20 action which is recognized as calcium channel blocking action and thus mediates apoptosis by blocking intracellular signaling and altering kinases activity [3]. It induces cell lysis through complement-dependent and independent mechanisms and also has direct target action against podocytes which is independent of its B-cell action [4,5]. Here it binds to sphingomyelin phosphodiesterase acid-like $3 \mathrm{~b}$ protein (SMPDL-3b) and regulates acid sphingomyelinase activity to prevent protein disruption of the actin cytoskeleton and podocyte apoptosis.

\section{Rituximab in ANCA Vasculitis}

Rituximab is now FDA approved drug for vasculitis.

Till date guidelines recommends cyclophosphamide as the first line therapy for crescentic small vessel pauciimune ANCA vasculitis, which is there for more than four decades now. With the success of multiple case reports, randomized trial were conducted which showed it as non-inferior and safer to cyclophosphamide, when used as an induction therapy. It has

Even showed superior results as compared to azathioprine when used as maintenance drug. Here below discussing briefly the results of these landmark studies which began new era for rituximab in ANCA vasculitis. 


\section{Rituxivas study}

Included 44 patients of new onset ANCA associated renal vasculitis. Rituximab on day $0,7,14$ and 21 was given with 2 doses of cyclophosphamide on day 0 and 14 in induction and compared with IV CYP and prednisolone therapy. No maintenance therapy was used. Results showed sustained remission of $76 \%$ in rituximab versus $82 \%$ in cyclophosphamide group at one year. No significant difference in the outcome of two therapies was noted [6].

\section{Clinical usage}

1. A dose of $375 / \mathrm{m}^{2} /$ intravenous infusion once weekly for 2-4 weeks. Dosage up to $1 \mathrm{~g}$ /infusion used.

2. Premedication with acetaminophen, antihistamine and steroid.

3. PCP prophylaxis for 3 months Drug Infusion requires preparation in normal saline (NS dilution half of dose strength) Initial rate of infusion is $50 \mathrm{mg} /$ hour, which in the absence of infusion toxicity increases to $50 \mathrm{mg} / \mathrm{hour}$ every 30 minutes. (Recommended by manufacturer with the maximum rate of $400 \mathrm{mg} /$ hour).

4. Monitoring advised with serum level of lymphocyte counts, proteinuria, circulating $\mathrm{B}$ cells (CD19<1\%), B cell recovery is usually detectable by 6 to 9 months.

5. Available in $100 \mathrm{mg} / 10 \mathrm{ml}$ and $500 \mathrm{mg} / 50 \mathrm{ml}$ strength.

6. Infusion related allergic reactions can be seen with first infusion, varying from mild fever, chills, headache and nausea to severe bronchospasm, hypotension and angioedema. Rarely severe muco-cutaneous reactions, ARDS, myocardial ischemia and arrhythmias.

\section{Rave trial}

RCT included 197 patients of new onset or relapsed ANCA associated vasculitis. Renal involvement was seen in $66 \%$ of enrolled patients. A weekly 4 dose of rituximab was compared with oral cyclophosphamide in induction and in maintenance no therapy in rituximab versus azathioprine in CYP group was compared. $64 \%$ in rituximab and $53 \%$ in cyclophosphamide group at 6 months achieved remission and subsequent remission in two groups seen at 12 and 18 months were $48 \mathrm{vs}$. $39 \%$ and 39 vs. $33 \%$ respectively. In relapsing disease $67 \%$ vs. $42 \%$ remission in rituximab and cyclophosphamide group at 12 month were documented respectively and suggested better outcome [7].

\section{Mainritsan trial}

French vasculitis group compared rituximab and azathioprine in the maintenance phase when the induction therapy used for two groups were cyclophosphamide and steroids. 118 patients were enrolled, $70 \%$ had renal involvement. Rituximab was given on day 0,14 and 3 doses at 6 monthly intervals. At 28 months, $29 \%$ relapses seen in azathioprine while $5 \%$ in rituximab group while both group had similar rates of adverse events [8]. A retrospective analysis of 172 patients with ANCA vasculitis suggested successful remission on maintenance rituximab therapy every 4 monthly for 7 year [9].

In eosinophillic granulomatous polyangitis, available limited data suggests good efficacy in achieving remission [10,11]. Further studies required establishing recommendations here.

\section{Recommendations}

KDIGO guidelines 2012, recommend (For Wegner granulomatous, microscopic polyangitis ) rituximab therapy as an:

1. Alternative second line induction therapy in necrotizing $G N$.

2. Alternative therapy in resistant and progressive diseases along with other options [12].

\section{EULAR/EDTA Guidelines 2015 for ANCA vasculitis:}

1. Best response in wegnesrgranulomatosis, microscopic polyangitis then eosinophillic granulomatous polyangitis.

2. For remission-induction of new-onset organ threatening or life-threatening or relapsing AAV.

3. Recommend treatment with a combination of glucocorticoids and either cyclophosphamide or rituximab.

4. Rituximab also recommended as an alternative in maintenance therapy [13].

\section{Rituximab in Lupus Nephritis}

Role of rituximab trial came with failure of the first line therapies in biopsy-proven active Class III, IV and V lupus nephritis (LN) as per International Society of Nephrology/Renal Pathology Society (ISN/RPS). Persistence of high activity is indicated clinically by microscopic hematuria, proteinuria and rise in creatinine and histo-pathologically by glomerular hypercellularity, cellular crescents, leukocytes infiltration, wire loops, fibrinoid necrosis and tubular mono-nuclear infiltration.

Despite initial reports of successful usage of rituximab in lupus nephritis, controversies still exists on its efficacy here. Both EXPLOR (for moderate to severe SLE) and LUNAR study (for Lupus nephritis) was expected to come with favorable outcome but results did not favor the primary outcome [14-16].

\section{Lunar}

RCT phase III trials enrolled 144 patients with class III or class IV LN to receive rituximab $(1,000 \mathrm{mg})$ or placebo on days 1,15 , 168 , and 182 . The primary end point was renal response outcome at week 52. (Complete and partial) renal response rates were $45.8 \%$ in placebo and $56.9 \%$ in receiving rituximab group $(P=0.18)$. Rate of serious adverse events, including infections, were similar in both groups (placebo versus rituximab) but neutropenia, leukopenia, and hypotension occurred more frequently in the rituximab group. Contrary to LUNAR trial, extended report of prospective observational study RITUXILUP (Rituximab and Mycophenolate mofetil without Oral Steroids for Lupus Nephritis) suggested successful outcome with complete and partial remission in $45 / 50$ patients, relapses 
in 11/50 and systemic flare in 6 patients over follow up of 65 weeks [17].

Another report in favor of rituximab role in refractory LN came from systematic analysis by Weidenbusch et al. involving 300 patients with a mean follow-up of 60 weeks in 26 studies. Both complete and partial remission was seen in $87 \%$ of patients with LN class III, $76 \%$ with class IV and $67 \%$ with Class V while complete responses alone was seen in $60 \%, 45 \%, 40 \%$ and $24 \%$ of class III, IV, V and mixed respectively in refractory lupus [18]. Dilemma still exists for its role and future trial may provide the definitive answer here [19].

RING: "Rituximab for Lupus Nephritis with Remission as a Goal” Clinical Trials.gov NCT01673295

\section{Recommendations}

1. KDIGO 2012 lupus nephritis [12].

2. In resistant lupus nephritis with either of other alternatives option like IgG.

3. ACR guidelines [18].

4. Rituximab -an alternative for resistant and refractory lupus nephritis

\section{Rituximb in anti-GBM}

No definitive data available. Disease often presents as rapidly progressive crescentic glomerulonephritis and if not respond to plasmapheresis and first line immunosuppressive treatment then progress to chronic kidney diseases. Case reports have shown successful outcome of B-cell therapy in refractory illness in term of disease activity but had no effect on improvement in GFR [19-21].

\section{Rituximab in membrano-proliferative GN}

Pilot study by Dillon et al. showed some benefit of Rituximab in type 1 MPGN. 2/6 had complete remission and $3 / 6$ had partial remission and mean time to achieve remission was 6-9 month [22]. Case series, and RCT in mixed cryoglobulinemia (MC) have shown significant improvement in proteinuria [23,24]. Multicenter study retrospectively evaluated the effects of rituximab in 87 patients with active $M C$ and 38/87 with Nephropathy. 92\% had association with HCV. 95\% showed significant improvement in proteinuria and $50 \%$ had complete remission [25]. In study of 93 patients by Saadoun et al. pegylated-interferon- $\alpha /$ ribavirin with rituximab was compared with Peg-IFN- $\alpha /$ Rbv alone. Clinical remission was achieved earlier in ritux group ( 5 vs. 8 months) and significant difference in renal response rates (80 vs.40\%) [26].

\section{Recommendation}

KDIGO [2D] suggest use of rituximab or other alternative for patients with HCV and mixed cryoglobulinemia (IgG/IgM) with nephrotic proteinuria or evidence of progressive kidney disease or an acute flare of cryoglobulinemia.

\section{Rituximab in IgA nephropathy}

1. No defined role till date. STOP- IgA study -an open label

2. RCT failed to show any benefit by adding rituximab $[27,28]$.

\section{Belimumab}

Approved by the Food and Drug Administration in 2011 for the treatment of active SLE but not for severe CNS lupus and severe nephritis. It is a human IgG1 $\lambda$ monoclonal antibody with elimination half-life of 19.4 days. Onset of action for B cell suppression is 8 weeks, with clinical manifestations improvement seen in 16 weeks [29]. Drug act by binding to the soluble form of B-lymphocyte stimulator (BlyS), which is a TNF superfamily ligand and thus block autoantibodies. It acts in the bone marrow and block plasma cell, which in turn inhibit prolonged antibody memory. It blocks intracellular signaling cascade through all three BlyS-binding receptors (trans membrane activator and CAML (calcium modulating cyclophilin ligand) interactor (TACI), BCMA and BAFF-R and finally $B$ cell proliferation. Belimumab also blocks $T$ cell secretion of interferon-gamma (INF- $\gamma$ ), interluekin-2 (IL-2) [IL-2-dependent proliferation of $T$ cells and antibody production] and also transition of immature $B$ cells from the $T 1$ to $T 2$ stage thus preventing mature $B$ cells formation in spleen [30].

\section{Clinical usage}

Dosage: $10 \mathrm{mg} / \mathrm{kg}$ IV injection on Days 0,14 and 28, and then every 4 weeks. For infusion, dilution in in $250 \mathrm{ml} 0.9 \%$ normal saline and administration in 1 hour. Premedication prophylaxis with anti-allergic, anti-inflammatory and hydrocortisone is recommended Monitoring with SLE activity markers.

Benalysta: $120 / 1.5 \mathrm{ml}$ and $400 \mathrm{mg} / 4.8 \mathrm{ml}$ sterile water to make a concentration of $80 \mathrm{mg} / \mathrm{ml}$. Costs of $\$ 443$ for $120 \mathrm{mg}$ and $\$ 1,477$ for $400 \mathrm{mg}$ vial. Infusion related reactions common. Especially with concomitant administration of mycophenolatemofetil. More than 5\% reported nausea, diarrhea, pyrexia, nasopharyngitis, bronchitis,insomnia, pain in extremity, depression, migraine, and pharyngitis.

\section{Belimumab in lupus erythromatosus}

Randomized double blind controlled trial in phase III now have shown its efficacy in lupus activity [31,32]. In BLISS-52, 865 patients' were enrolled and efficacy was analyzed at week 52 . $58 \%$ vs. $44 \%$ remission rate seen with belimumab and placebo respectively.

BLISS-76, 819 SLE patients were analyzed at week 52 and then at week 76 and reduced disease activity was noted $43 \%$ and $34 \%$ respectively. Efficacy noted at week 76 was not statistically significant. In future, results of two new trials in SLE patients with nephritis may give definitive role of Belimumab in lupus nephritis and change present treatment guidelines. The two trials are currently recruiting lupus nephritis patients. NCT02260934: (CALIBRATE STUDY) Rituximab Plus Cyclophosphamide Followed by Belimumab for the Treatment of Lupus Nephritis (ITN055AI). NCT01639339: (BLISS-LN): Efficacy 
and Safety of Belimumab in Patients With Active Lupus Nephritis. Other than SLE nephritis, Belimumab is now being evaluated for the treatment in ANCA vasculitis (BREVAS study, NCT01663623).

\section{Recommendation}

Belimumab: FDA approval for SLE but no recommendation for active CNS lupus and lupus nephritis.

\section{Eculizumab}

It is a long-acting humanized monoclonal $\operatorname{lgG} 2 / 4 \mathrm{~K}$ antibody against complement $\mathrm{C} 5$. Half-life approximately $11 \pm 3$ days and is mainly distributed in the vascular space. It binds to complement $\mathrm{C} 5$ and prevent its breakdown into $\mathrm{C} 5 \mathrm{a}$ and $\mathrm{C} 5 \mathrm{~b}$ by C5 convertase. In activated complement pathway, complement C5a increases the permeability of blood vessels and attracts inflammatory cells by chemo-taxis while $\mathrm{C} 5 \mathrm{~b}$ binds to other complement components (C6, C7 and C8) to form the MAC (membrane attack complex) [33,34]. MAC has role in killing the microorganism especially Neisseria. First successful role came in paroxysmal nocturne al hemoglobinuria and now approved for atypical HUS [35].

\section{Eculizumab in complement mediated glomerulonephritis and aHUS}

C3 glomerulopathy is recently coined term for the disease process due to abnormal activation of complement pathway. It is further classified into dense deposit disease (DDD) and C3 glomerulonephritis. Understanding pathophysiology and complement activation is beyond the scope of this review but after knowing this complex physiology of complement system activation and its approval in aHUS, multiple cases were reported for its role in MPGN [36,37]. Both beneficial as well as failure reports came out and also successful withdrawal from dialysis after drug initiation reported [38-40]. Bomback et al. demonstrated successful outcome of Eculizumab in six patients (three patients each with dense deposit disease and C3 glomerulonephritis) in term of proteinuria and improved renal profile. Even repeat renal biopsy after 1 year suggest less activity with no evidence of endocapillary proliferation [41]. Currently two open label studies in phase I (NCT01221181) and phase II (NCT02093533) are undergoing and their outcome shall provide further information regarding efficacy and timing of initiation [42].

\section{Eculizumab in Atypical Hemolytic uremic syndrome}

Characterized by activation of complement mediated hemolysis and renal syndrome. Eculizumab is FDA approved for aHUS. Multi-centric study in phase II has reported its efficacy in aHUS $[43,44]$. Phase II study in trial 1 , enrolled 17 patients with progressing thrombotic microangiopathy (TMA). Hematologic normalization was achieved in $76 \%$ at 26 weeks and $88 \%$ at 1 year. Complete TMA response was achieved by $65 \%$ at week 26 and $76 \%$ at the 1 and 2 -year. $88 \%$ had TMA free events. Trial II, in phase II study, 20 patients with long duration of aHUS and chronic kidney disease were enrolled. TMA free events achieved at 26 weeks 1 and 2 year were 80\%, 85\% and 95\% respectively. 18 patients (90\%) achieved and maintained hematological remission. Complete TMA response was achieved in $25 \%$ at 26 weeks, $35 \%$ at 1 year, and $55 \%$ at the 2 year. None of the patients in study had meningococcal infection and no graft loss seen in renal transplant patient with eculizumab therapy. Previously, French study group reviewed and summarized case reports of 24 patients including 11 children, where eculizumab was used as a curative therapy in native kidneys $(n=14)$ or transplanted kidneys with HUS $(n=10)$. Among these 24 patients, $15(62.5 \%)$ were found to have complement mutations, mostly in $\mathrm{CFH}(10 / 24,42 \%)$. Excellent outcome was noted in both pediatric and adult patients [45]. Another aHUS report gave some hope in deciding duration and cost burden of therapy where drug was discontinued for 95 months in 10 patients who had achieved stable remission. In this report 3 patients relapsed within 6 weeks of stopping therapy. 7/10 did well and no other adverse effect noted [46].

\section{Clinical usage}

1. Dose variable as per weight (10-40 kg and more) 300 to 900 mg per week for 1-4 weeks then 300-1200 mg every 2-3 weeks.

2. Infusion $300 \mathrm{mg} / 30 \mathrm{ml}$ in $30 \mathrm{ml}$ dilution (NS/ Dextrose $5 \%$, ringer lactate) and transfuse over $35 \mathrm{~min}$.

3. Monitoring with HUS activity markers for 8-12 weeks if stopped.

4. Meningococcal vaccination 2 weeks prior is recommended

5. Soliris : $10 \mathrm{mg} / \mathrm{ml}$ solution for intravenous infusion. Available strength: $30 \mathrm{ml} / 300 \mathrm{mg}$ single use vial. Cost is $\$ 6830$ per $300 \mathrm{mg}$ vial.

6. Risk of meningococcal infection, and serious infections due to Streptococcus pneumoniaeandH. influenza type b (Hib). Prior vaccines are recommended.

7. No infusion related reactions noted.

8. Adverse effects $(<15 \%)$ in trial. Include hypertension, URTI, diarrhea, headache, anemia, vomiting, nausea, UTI andleukopenia. Acceleration of hepatotoxicity.

\section{Brief on other monoclonal antibodies}

Multiple monoclonals have been introduced in immune mediated glomerular disease. Action on specific target receptors, make them more specific with less side effects compared to previous available therapies. Target receptor can be at $T$ cell (Alemtuzumab, Basiliximab), B-cell (Epratuzumab, Belimumab), Anti-cytokine (infliximab, adalimumab), complement (CCX168) and co stimulation (Abatacept, belatacept) pathway. Few with successful efficacy in GN have been discussed above. Many other monoclonal antibodies were studied in nephritis and a long list of monoclonal failed to show benefit. ACCESS study reported that abatacept/CTLAA-4-Ig (Cytotoxic T-lymphocyte-associated antigen 4-immunoglobulin fusion protein) plus low dose intravenous cyclophosphamide followed by azathioprine did not improve remission rates in patients with lupus nephritis [47]. Small open label trial assessed 
the safety and efficacy of abatacept as a remission therapy in patients with relapsing, non severe GPA did not provide statistical significant result [48]. A phase III, multicenter RCT to assess the efficacy of abatacept to permit steroid free remission in relapsing GPA is underway. Abatacept is an inhibitor of the Tcell co-stimulatory signaling molecule B7-1 (CD80) and CD86 that are present on the surface of $B$ cells, dendritic cells, and macrophages. Ocrelizumab, anti CD-20 failed show significant much benefit in Lupus nephritis and trials terminated due to association with very high rate of infection [49]. Anti-IL6 and IL$6 R$ are now being tested in lupus nephritis. A phase II trial of sirukumab (an antill6 monoclonal antibody) in lupus nephritis reported negative findings, with a high incidence of infectious complications [50]. A small study of tocilizumab (an antilL6R monoclonal antibody) in SLE nephritis yet to give result. Anticytokine therapy, certolizumab, pegol, and golimumab have yet to show their effect in vasculitis. Mepolizumab is an antilL5 monoclonal antibody that according to case reports showed favorable response in patients with EGPA [51,52]. Two RCTs are assessing the efficacy of mepolizumab induced remission and steroid sparing capacity in EGPA [5355]. Omalizumab- an antilgE monoclonal antibody that prevents IgE binding to Fce receptors on mast cells, studied in case series but yet show its definitive results in EGPA [56]. Apart from glomerulonephritis, monoclonals have been used extensively in kidney transplantation and nephrotic syndrome. Rituximab has successful outcome in calcineurin dependent, membranous nephropathy, steroid dependent Nephrotic syndrome. Also Other monoclonals like Abatacept, Fresolimumab (an IgG4 humanized Monoclonal antibody against all three isoforms of TGF-b), Adalimumab (human monoclonal IgG antibody against tumor necrosis factor $\alpha$ (TNF- $\alpha)$ have shown significant reduction in proteinuria and given some hope in FONT (First portion of novel therapies) study for focal segmental glomerulosclerosis [57].

\section{Recommendation}

Eculizumab: FDA approval for aHUS but yet to see definite outcome and recommendations in C3 glomerulopathy nephritis.

\section{Conclusion}

Monoclonal antibodies are emerging with a great hope for refractory and primary renal diseases. This mini review was for glomerulonephritis and detailed discussion of their role in nephrotic syndrome and transplantation is beyond scope of this review. Although future of immune mediated kidney diseases lies with monoclonal antibodies, but cost is limitation till date. Efficacy and safety both have been shown in glomerulonephritis and with more data results; we shall be able to have their superior role in guidelines.

\section{References}

1. Tauber Al (1992) The birth of immunology III. The fate of the phagocytosis theory. Cell Immunol 139: 505-530.
2. Marks LV (2013) A healthcare revolution in the making: the story of césar milstein and monoclonal antibodies: making monoclonal antibodies. WhatisBiotechnology.org.

3. Tedder TF, Engel $P$ (1994) CD20: a regulator of cell-cycle progression of B lymphocytes. Immunol Today 15: 450-454.

4. Perosa F, Favoino E, Caragnano MA, Dammacco F (2006) Generation of biologically active linear and cyclic peptides has revealed a unique fine specificity of rituximab and its possible cross-reactivity with acid sphingomyelinase-like phosphodiesterase 3b precursor. Blood 107: 1070-1077.

5. Fornoni J, Wei SC, Merscher-Gomez S, Aguillon-Prada R, Jauregui AN, et al. (2011) Rituximab targets podocytes in recurrent focal segmental glomerulosclerosis. Sci. Transl. Med. 3: 46.

6. Stone JH, Merkel PA, Spiera R, Seo P, Langford CA, et al. (2010) Rituximab versus cyclophosphamide for ANCA-associated vasculitis. N Engl J Med 363: 221-232.

7. Jones RB, Tervaert JW, Hauser T, Luqmani R, Morgan MD, et al. (2010) Rituximab versus cyclophosphamide in ANCA-associated renal vasculitis. N Engl J Med 363: 211-220.

8. Guillevin L, Pagnoux C, Karras A, Khouatra C, Aumaître O, et al. (2014) Rituximab versus azathioprine for maintenance in ANCAassociated vasculitis. N Engl J Med 371: 1771-1780.

9. Pendergraft WF 3rd, Cortazar FB, Wenger J, Murphy AP, Rhee EP, et al. (2014) Long-term maintenance therapy using rituximabinduced continuous B-cell depletion in patients with ANCA vasculitis. Clin J Am Soc Nephrol 9: 736-744.

10. Muñoz SA, Gandino IJ, Orden AO, Allievi A (2015) Rituximab in the treatment of eosinophilic granulomatosis with polyangiitis. Reumatol Clin 11: 165-169.

11. Mohammad AJ, Hot A, Arndt F, Moosig F, Guerry MJ, et al. (2016) Rituximab for the treatment of eosinophilic granulomatosis with polyangiitis (Churg-Strauss). Ann Rheum Dis 75: 396-401.

12. Kidney Disease (2012) Improving Global Outcomes (KDIGO) Glomerulonephritis Work Group. KDIGO clinical practice guideline for glo- merulonephritis. Kidney Int Suppl 2: 139-274.

13. Melander C, Salle'e M, Trolliet P (2009) Rituximab in severe lupus nephritis: Early B- cell depletion affects long-term renal outcome. Clin J Am Soc Nephrol 4: 579-587

14. Merrill JT (2010) Efficacy and safety of rituximab in moderately-toseverely active systemic lupus erythematosus: the randomized, double-blind, phase II/III systemic lupus erythematosus evaluation of rituximab trial. Arthritis Rheum 62: 222- 233.

15. Condon MB, Ashby D, Pepper RJ, Cook HT, Levy JB, et al. (2013) Prospective observational single-centre cohort study to evaluate the effectiveness of treating lupus nephritis with ri- tuximab and mycophenolate mofetil but no oral steroids. Ann Rheum Dis 72: $1280-1286$.

16. Weidenbusch M, Römmele C, Schröttle A, Anders HJ (2013) Beyond the LUNAR trial. Efficacy of rituximab in refractory lupus nephritis. Nephrol Dial Transplant 28: 106-111.

17. Rovin BH (2012) Efficacy and safety of rituximab in patients with active proliferative lupus nephritis: the lupus nephritis assessment with rituximab study. Arthritis Rheum 64: 1215-1226.

18. Gunnarsson I, Jonsdottir T (2013) Rituximab treatment in lupus nephritis - where do we stand? Lupus 22: 381-389.

19. Hahn BH (2012) American College of Rheumatology guidelines for screening, treatment, and management of lupus nephritis. Arthritis Care Res (Hoboken) 64: 797-808. 
20. Arzoo K, Sadeghi S, Liebman HA (2002) Treatment of refractory antibody mediated autoimmune disorders with an anti-CD20 monoclonal antibody (rituximab). Ann Rheum Dis 61: 922-924.

21. Shah Y, Mohiuddin A, Sluman C, Daryanani I, Ledson T, et al. (2012) Rituximab in anti-glomerular basement membrane disease. QJM 105: 195-197.

22. Touzot M, Poisson J, Faguer S, Ribes D, Cohen P, et al. (2015) Rituximab in anti-GBM disease: A retrospective study of 8 patients. J Autoimmun 60: 74-79.

23. Dillon JJ, Hladunewich M, Haley WE, Reich HN, Cattran DC, et al. (2012) Rituximab therapy for Type I membranoproliferative glomerulonephritis. Clin Nephrol 77: 290-295.

24. Korte MR, van Heerde MJ, de Man RA, Betjes MH (2008) Rituximab for the treatment of glomerulonephritis in hepatitis $C$ associated cryoglobulinaemia. Neth J Med 66: 27-30.

25. [da Silva Fucuta Pereira P, Lemos LB, de Oliveira Uehara SN, de Souza $E$, et al. (2009) Long-term efficacy of rituximab in hepatitis $C$ virus-associated cryoglobulinemia. Rheumatol Int 30: 1515-1518.

26. Ferri C (2011) Treatment with rituximab in patients with mixed cryoglobulinemia syndrome: Results of multicenter cohort study and review of the literature, Autoimmun Rev.

27. Saadoun D, Resche Rigon M, Sene D, Terrier B, Karras A, et al (2010) Rituximab plus Peg-interferon-a/ribavirin compared with Peg-interferon-a/ribavirin in hepatitis C-re- lated mixed cryoglobulinemia. Blood 116: 326-334.

28. Chancharoenthana W, Townamchai N, Leelahavanichkul A, Wattanatorn T, Kanjanabuch T, et al. (2016) Rituximab for recurrent iga nephropathy in kidney transplantation: a report of 3 cases and proposed mechanisms. Nephrology (Carlton) 12: 122-127.

29. Thomas R, Frank, ChristinaFitzner F, Claudia S, Martin Z, et al. (2015) Intensive supportive care plus immunosuppression in iga nephropathy, STOP IgAN. N Engl J Med Dec. 2015; 373:2225-36.

30. Belimumab DC (2008) an anti-BLyS human monoclonal antibody for potential treatment of inflammatory autoimmune diseases. Expert opinion on biological therapy 8: 1805-1814.

31. Wiglesworth AK, Ennis KM, Kockler DR (2010) Belimumab: a BLySspecific inhibitor for systemic lupus erythematosus. Annals of Pharmacotherapy. 2010 Dec 44: 1955-1961.

32. Merrill JT, Ginzler EM, Wallace DJ, McKay JD, Lisse JR, et al. (2012) LBSL02/99 Study Group. Long-term safety profile of belimumab plus standard therapy in patients with systemic lupus erythematosus. Arthritis Rheum 64: 3364-3373.

33. Navarra SV, Guzmán RM, Gallacher AE, Hall S, Levy RA, et al. (2011) Efficacy and safety of belimumab in patients with active systemic lupus erythematosus: a randomised, placebo-controlled, phase 3 trial. Lancet 377: 721-731.

34. Manrique J, Cravedi P (2014) Role of monoclonal antibodies in the treatment of immune-mediated glomerular diseases. Nefrologia 34: 388-397.

35. Campistol JM, Arias M, Ariceta G (2013) An update for atypical haemolyticuraemic syndrome: diagnosis and treatment. $A$ consensus document. Nefrologia 33: 27-45.

36. Rousset-Rouvière C, Cailliez M, Garaix F (2014) Rituximab fails where eculizumab restores renal function in C3nef-related DDD. PediatrNephrol 2014; 29: 1107-1111.
37. Le Quintrec M, Lionet A, Kandel C, Bourdon F, Gnemmi V, et al. (2015) Eculizumab for treatment of rapidly progressive C3 glomerulopathy. Am J Kidney Dis 65: 484-489.

38. Vivarelli M, Pasini A, Emma F (2012) Eculizumab for the treatment of dense-deposit disease. new englandjournal of medicine 366: 1163-1165.

39. Inman M, Prater G, Fatima H, Wallace E (2015) Eculizumabinduced reversal of dialysis-dependent kidney failure from C3 glomerulonephritis. Clin Kidney J 8: 445-448.

40. Bomback AS, Smith RJ, Barile GR, Zhang Y, Heher EC, et al. (2012) Eculizumab for dense deposit disease and C3 glomerulonephritis. Clin J Am Soc Nephrol 7: 748-756.

41. Rodriguez-Osorio L, Ortiz A (2015) Timing of eculizumab therapy for C3 glomerulonephritis. Clin Kidney J 8: 449-452.

42. Licht C, Greenbaum LA, Muus P, Babu S, Bedrosian CL, et al. (2015) Efficacy and safety of eculizumab in atypical hemolytic uremic syndrome from 2-year extensions of phase 2 studies Kidney International 87: 1061-1073.

43. Legendre CM, Babu S, Furman RR (2010) Safety and efficacy of eculizumab in ahus patients resistant to plasma therapy: interim analysis from a phase ii trial. J Am Soc Nephrol 21: 402.

44. Zuber J, Fakhouri F, Roumenina LT, Loirat C, Fre'meaux-Bacchi V, et al. (2012) Use of eculizumab for atypical haemolytic uraemic syndrome and C3 glomerulopathies. Nat. Rev. Nephrol 8: 643-657.

45. Ardissino G, Testa S, Possenti I, Tel F, Paglialonga F, et al. (2014) Discontinuation of eculizumab maintenance treatment for atypical hemolytic uremic syndrome: a report of 10 cases. Am J Kidney Dis 64: 633-637.

46. Aringer M, Smolen JS (2012) Therapeutic blockade of TNF in patients with SLE-promising or crazy? Autoimmun Rev 11: 321-325.

47. US National Libary of Science (2013) ClinicalTrials.gov [online], http://www.clinicaltrials.gov/ct2/show/ NCT00447265.

48. US National Libary of Science (2009) ClinicalTrials.gov [online], http://www.clinicaltrials.gov/ct2/show/ NCT00368264.

49. Zheng B, Yu XQ, Greth W, Robbie GJ (2015) Population pharmacokinetic analysis of sifalimumab from a clinical Phase IIb trial in systemic lupus erythematosus patients. Br. J. Clin. Pharmacol.

50. Kahn JE, Grandpeix-Guyodo C, Marroun I, Catherinot E, Mellot F, et al. (2010) Sustained response to mepolizumab in refractory Churg-Strauss syndrome. J Allergy Clin Immunol 125: 267-270.

51. Kim S, Marigowda G, Oren E, Israel E, Wechsler ME (2010) Mepolizumab as a steroid-sparing treatment option in patients with Churg-Strauss syndrome. J. Allergy Clin. Immunol 125: 13361343.

52. Herrmann K, Gross W L, Moosig F (2012) Extended follow-up after stopping mepolizumab in relapsing/ refractory Churg-Strauss syndrome. Clin. Exp. Rheumatol 30: S62-65.

53. Matthews LT, Sibeko S, Mansoor LE, Yende-Zuma N, Bangsberg DR, et al. (2013) Women with pregnancies had lower adherence to $1 \%$ tenofovir vaginal gel as HIV preexposure prophylaxis in CAPRISA 004, a phase IIB randomized-controlled trial. PLoS One 8: e56400.

54. US National Libary of Science (2012) ClinicalTrials.gov [online], http://www.clinicaltrials.gov/ct2/show/ NCT00716651.

55. Iglesias E (2014) Successful management of Churg-Strauss syndrome using omalizumab as adjuvant immunomodulatory 
therapy: first documented pediatric case. Pediatr. Pulmonol 49: E78-E81.

56. Trachtman H, Fervenza FC, Gipson DS, Heering P, Jayne DR, et al. (2011) A phase 1, single-dose study of fresolimumab, an anti-TGFb antibody, in treatment-resistant primary focal segmental glomerulosclerosis. Kidney Int 79: 1236-1243.
57. Joy MS, Gipson DS, Powell L, MacHardy J, Jennette JC, et al. (2010) Phase 1 trial of adalimumab in focal segmental glomerulosclerosis (FSGS): II. Report of the FONT (Novel Therapies for Resistant FSGS) study group. Am J Kidney Dis 55: 50-60. 